\title{
ResearchGate
}

See discussions, stats, and author profiles for this publication at: http://www.researchgate. net/publication/282249452

\section{The chemical stability and cytotoxicity of carbonyl iron particles grafted with poly(glycidyl methacrylate) and the magnetorheological activity of their suspensions $\dagger$}

ARTICLE in RSC ADVANCES · AUGUST 2015

Impact Factor: $3.84 \cdot$ DOI: 10.1039/c5ra11968e

READS

23

8 AUTHORS, INCLUDING:

Miroslav Mrlík

Tomas Bata University in Zlín 42 PUBLICATIONS 105 CITATIONS

SEE PROFILE

\section{Petr Humpolícek}

Tomas Bata University in Zlín 54 PUBLICATIONS 158 CITATIONS

SEE PROFILE
Jaroslav Mosnacek

Slovak Academy of Sciences

71 PUBLICATIONS 428 CITATIONS

SEE PROFILE

Vladimír Pavlínek

Tomas Bata University in Zlín 103 PUBLICATIONS 889 CITATIONS

SEE PROFILE 


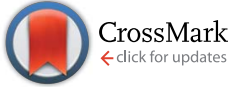

Cite this: RSC Adv., 2015, 5, 72816
Received 21st June 2015

Accepted 21st August 2015

DOI: 10.1039/c5ra11968e

www.rsc.org/advances

\section{The chemical stability and cytotoxicity of carbonyl iron particles grafted with poly(glycidyl methacrylate) and the magnetorheological activity of their suspensions $\dagger$}

\begin{abstract}
Martin Cvek, ${ }^{\text {ab }}$ Miroslav Mrlík, ${ }^{\text {*ac }}$ Markéta llčíková, ${ }^{\text {cd }}$ Jaroslav Mosnáček, ${ }^{d}$ Vladimir Babayan, ${ }^{a}$ Zdenka Kuceková, ${ }^{a}$ Petr Humpolíček ${ }^{a}$ and Vladimir Pavlínek ${ }^{a}$

Carbonyl iron ( $\mathrm{Cl}$ ) particles were grafted with poly (glycidyl methacrylate) (PGMA) using atom transfer radical polymerization. Compact coating of PGMA largely improved the chemical stability of the particles in an acid environment and thus reduced the common drawback of bare $\mathrm{Cl}$ particles. Furthermore, due to possible medical applications of $\mathrm{Cl}$-polymer systems for magnetic drug targeting, an in vitro cytotoxicity test was performed using an NIH/3T3 cell line. The cell viability was evaluated by spectrometric assay (MTT). The results show that the prepared particles are not cytotoxic. Moreover, bare $\mathrm{Cl}$ particles as well as synthesized core-shell particles were suspended in silicone oil, and the rheological behavior of MR suspensions was investigated in controlled shear rate mode under various magnetic field strengths. Dynamic yield stress as a measure of the rigidity of the created internal structures of the suspensions was determined using the Herschel-Bulkley model, which provided a reasonably good fit for rheological data. MR suspensions of PGMA-coated particles exhibited only slightly decreased yield stresses due to their negligibly-affected magnetic performance.
\end{abstract}

\section{Introduction}

Magneto-responsive magnetorheological (MR) suspensions composed of soft ferromagnetic particles $0.1-10 \mu \mathrm{m}$ in size dispersed in non-magnetic carrier liquids exhibit remarkable rheological behavior including rapid, reversible and tunable transitions from liquid-like to solid-like states under an applied magnetic field..$^{1-4}$ Drastic changes of physical properties, namely viscosity, yield stress or shear modulus occur within a few milliseconds after the application of the magnetic field due to the alignment of dispersed particles into column-like structures, which is a process based on the magnetic field-induced polarization of the particles. ${ }^{5-7}$ This phenomenon makes MR suspensions desirable for a significant number of engineering applications incorporating damping systems, ${ }^{8-10}$ torque transducers, ${ }^{11}$ the finishing of optics, ${ }^{12}$ or robotics. ${ }^{13}$

${ }^{a}$ Centre of Polymer Systems, University Institute, Tomas Bata University in Zlin, Trida T. Bati 5678, 76001 Zlin, Czech Republic. E-mail: mrlik@cps.utb.cz

${ }^{b}$ Polymer Centre, Faculty of Technology, Tomas Bata University in Zlin, nam. T. G. Masaryka 275, 76272 Zlin, Czech Republic

${ }^{c}$ Center for Advanced Materials, Qatar University, P. O. Box 2713, Doha, Qatar ${ }^{d}$ Polymer Institute, Slovak Academy of Sciences, Dubravska cesta 9, 84541 Bratislava 45, Slovakia

$\dagger$ Electronic supplementary information (ESI) available. See DOI: 10.1039/c5ra11968e
However, poor thermo-oxidation and sedimentation stabilities are crucial problems of classical MR suspensions, which reduce their broader practical utilization. ${ }^{\mathbf{1 3 , 1 4}}$ Moreover, in many cases an overlooked drawback is also the poor chemical stability of bare CI particles in an acidic environment. Such drawbacks can be efficiently reduced by the modification of the particle surface with polymer chains, allowing for the involvement of core-shell structured particles in the preparation of MR suspensions. ${ }^{1-3,7,15,16}$ However, conventional coating methods tend to cause a large decrease in the magnetic permeability of the particles. Using surface-initiated reversible deactivation radical polymerization, such as atom transfer radical polymerization (ATRP), allows the precise control of the molecular weight of grafted polymer chains. This approach also enables control over the polymer layer thickness on an inorganic substrate since the thickness of the layer progressively increases with the molecular weight of the densely-grafted polymer chains. ${ }^{17}$ Therefore, such a method enables an adjustment to MR performance via the controlled thickness of the polymeric layer on the particles surface. ${ }^{\mathbf{1 8}}$

Generally, MR suspensions are in most cases subjected to shear flow while exhibiting nearly Newtonian behavior in the absence of a magnetic field (off-state), or develop a yield stress and shear rate-dependent apparent viscosity after the application of an external field (on-state). ${ }^{\mathbf{1 5 , 1 0}}$ The behavior of MR suspensions can be characterized by the Bingham plastic 
model, ${ }^{19}$ which belongs among the most popular models because of its simplicity and sufficient accuracy. ${ }^{20}$ Nevertheless, the on-state pseudoplastic behavior of MR suspensions is in contradiction to the Bingham model. Therefore some authors $^{21-23}$ have employed the Herschel-Bulkley (HB) model, which replaces constant plastic viscosity in the Bingham model with a shear rate-dependent power-law relation in order to include post-yield stress shear thinning characteristics.

In our preceding paper, ${ }^{18}$ we showed that the poly(glycidyl methacrylate) (PGMA) coating of CI particles via ATRP resulted in a considerable improvement of thermo-oxidation and sedimentation stabilities, while the response of the modified particles to a magnetic field remained at an acceptable level. However, in real-life applications, the anti-corrosion stability of the particles also plays an important role; for instance in the final polishing of high precision optics, a decrease in $\mathrm{pH}$ can improve the MR finishing of certain polycrystalline materials. ${ }^{24}$

The first part of this article deals with the examination of the chemical stability of PGMA-coated CI particles in an acidic environment in order to shift this material closer to potential real-life applications. Moreover, due to possible biomedical applications, ${ }^{25-28}$ attention is paid to an indicative in vitro cytotoxicity test, which was performed in order to analyze the basic cellular response to the tested substances. PGMA has recently gained interest in drug and biomolecule binding, ${ }^{29}$ and for this reason it was chosen as a coating layer for this study. In the second part, the magnetic performance of the bare and modified CI particles was investigated using the Jiles-Atherton (J-A) model, while their suspensions' rheological behavior was studied, and the obtained flow curves were fitted with the HB model in order to predict dynamic yield stresses and thus further evaluate yield stress development as a result of the polymer coating layer applied on the surface of CI particles.

\section{Experimental}

\section{Materials}

All chemicals were used as received. Carbonyl iron (CI) powder (SL grade) with a purity higher than $99.5 \%$ was obtained from BASF (Germany). Glycidyl methacrylate (GMA, 97\%), $\alpha$-bromo isobutyryl bromide (BiBB, 98\%), (3-aminopropyl) triethoxysilane (APTES, $\geq 98 \%$ ), ethyl $\alpha$-bromo isobutyrate (EBiB, 98\%), triethylamine $\left(\mathrm{Et}_{3} \mathrm{~N}, \geq 99 \%\right), N, N, N^{\prime}, N^{\prime \prime}, N^{\prime \prime}$-pentamethyldiethylenetriamine (PMDETA, $\geq 99 \%$ ), copper bromide (CuBr, $\geq 99 \%$ ), anisole (99\%) and aluminum oxide (neural, Brockmann I) were produced by Sigma Aldrich (USA). Other chemicals, namely tetrahydrofuran (THF, p.a.), acetone (p.a.), ethanol (absolute anhydrous, p.a.), toluene (p.a.), and hydrochloric acid ( $\mathrm{HCl}$, $35 \%$, p.a.) were purchased from Penta Labs (Czech Republic). MR suspensions were prepared using the silicone oil Lukosiol M200, which is a product of Chemical Works Kolín (Czech Republic; dynamic viscosity of $194 \mathrm{mPa}$ s, density of $0.97 \mathrm{~g} \mathrm{~cm}^{-3}, 25{ }^{\circ} \mathrm{C}$ ).

An NIH/3T3 mouse embryonic fibroblast cell line (ATCC CRL-1658), Dulbecco's Modified Eagle Medium, penicillin/ streptomycin (PAA Laboratories GmbH, Austria) and MTT (3- (4,5-dimethylthiazol-2-yl)-2,5-diphenyltetrazolium bromide) (Invitrogen Corporation, USA) were used for cytotoxicity tests.

CI particles grafted with PGMA chains (CI-PGMA) were prepared as described in a previous paper. ${ }^{18}$

\section{Characterization}

Monomer conversion during polymerization and the molecular weight of grafted polymer chains were characterized using proton nuclear magnetic resonance $\left({ }^{1} \mathrm{H}\right.$ NMR $)(400 \mathrm{MHz}$ VNMRS Varian NMR spectrometer, Varian, Japan) and gel permeation chromatography (GPC) (PL-GPC220 Agilent, Japan), respectively. The morphology and dimensions of the particles were observed using scanning electron microscopy (SEM) (Tescan Vega II LMU, Czech Republic). Modification of the CI surface with PGMA chains was proved by energy-dispersive spectroscopy (EDS) also performed on Tescan, and Fourier transform infrared spectroscopy (FTIR) (Nicolet 6700, USA) in the attenuated total reflectance (ATR) mode within the wavenumber range of $4000-500 \mathrm{~cm}^{-1}$ using a germanium crystal. The magnetic properties of both coated and bare CI particles were evaluated using a vibration sample magnetometry (VSM) (Model 7407, Vibrating Sample Magnetometer, Lakeshore, USA) employing a magnetic field strength of $\pm 870 \mathrm{kA} \mathrm{m}^{-1}$. The nonlinear magnetization curve of ferromagnetic particles can be described by the $\mathrm{J}-\mathrm{A}$ model: ${ }^{30}$

$$
M(H)=M_{\mathrm{S}}\left[\operatorname{coth}\left(\frac{H_{\mathrm{e}}}{A}\right)-\left(\frac{A}{H_{\mathrm{e}}}\right)\right]
$$

where $M$ denotes magnetization of the material, $H$ the magnetic field strength, and $M_{\mathrm{S}}$ the saturation magnetization; $A$ is the parameter describing magnetization shape without hysteresis, whereas $H_{\mathrm{e}}$ is the effective magnetic field strength given by:

$$
H_{\mathrm{e}}=H+\alpha M
$$

where $\alpha$ is the coefficient describing coupling between domains. Hence, obtained magnetization curves ${ }^{\mathbf{1 8}}$ are further analyzed in this paper. Moreover, the effect of polymer coating on the stability against sedimentation was studied with the help of a Tensiometer Krüss K100 (Krüss GmbH, Hamburg, Germany) at laboratory conditions. The measurements were performed on the MR suspensions based on $10 \mathrm{wt} \%$ particle concentration. Such concentration was chosen as an optional for the measurement with respect to the principle of tensiometric method.

\section{Chemical stability}

The anti-acid-corrosion stability of bare CI particles and both types of CI-PGMA particles were investigated. The amount of $1 \mathrm{~g}$ of appropriate particles was suspended in $20 \mathrm{~mL}$ of $0.05 \mathrm{M} \mathrm{HCl}$, and the $\mathrm{pH}$-value was measured as a function of time. The instrument (SensoDirect pH110, Tintometer GmbH, Germany) was previously calibrated using two standard buffers at laboratory temperature. The acidic suspensions were mechanically stirred during the experiment, while prior to each measurement 
the probe of the $\mathrm{pH}$-meter was cleaned by rinsing with distilled water and dried.

\section{Cytotoxicity}

Determination of cytotoxicity was carried out according to the international standard EN ISO 10993-5 using an NIH/3T3 mouse embryonic fibroblast cell line (ATCC, CRL-1658), which is a commonly used cell line for cytotoxicity testing of materials. $^{31-34}$ As a culture medium, Dulbecco's Modified Eagle Medium - high glucose, added $10 \%$ calf serum and penicillin/ streptomycin, $100 \mu \mathrm{g} \mathrm{mL} \mathrm{m}^{-1}$ (PAA Laboratories $\mathrm{GmbH}$, Austria) - was used.

Extract preparation. Prior to extraction, the samples were disinfected at $120^{\circ} \mathrm{C}$ for 40 minutes (Binder ED 53, Germany). Tested samples - bare CI particles and CI-PGMA-1 particles were extracted according to ISO 10993-12 in the amount of $0.2 \mathrm{~g}$ of powder per $1 \mathrm{~mL}$ of culture medium and incubated at $(37 \pm 1)$ ${ }^{\circ} \mathrm{C}$ under stirring for $(24 \pm 1) \mathrm{h}$. The parent extracts $(100 \%)$ were diluted in the culture medium in order to obtain extracts of concentrations 75, 50, 25, 10 and 1 vol\%. The extracts were used for cytotoxicity testing within $24 \mathrm{~h}$.

Cytotoxicity evaluation. The cells were seeded on microtitration plates (TPP, Switzerland) in a concentration of $1 \times 10^{5}$ per $\mathrm{mL}$ and pre-cultivated for $24 \mathrm{~h}$. The culture medium was subsequently replaced with appropriate extracts. The cells were cultivated for $24 \mathrm{~h}$ in the presence of individual extracts. The cell viability was evaluated by MTT assay (Invitrogen Corporation, USA). The absorbance was measured at $570 \mathrm{~nm}$ with an Infinite M200 Pro NanoQuant absorbance reader (Tecan, Switzerland). All the tests were performed in quadruplicates. Cell viability was expressed as the percentage of cells present in the corresponding extract relative to cells cultivated in a pure growth medium (reference, $100 \%$ viability). Values $>0.8$ were assigned to no cytotoxicity, $0.6-0.8$ to mild cytotoxicity, $0.4-0.6$ to moderate cytotoxicity, and $<0.4$ to severe cytotoxicity. The morphology of cells was observed by an inverted Olympus phase contrast microscope (Olympus IX81, Japan).

\section{Sample preparation and rheological properties in steady shear} flow

Bare CI particles as well as both types of CI-PGMA were suspended in silicone oil ( $40 \mathrm{wt} \%$ of particles in oil). In order to avoid settling and provide homogeneous samples, the MR suspensions were thoroughly stirred before each measurement. Raw rheological data was measured with a rotational rheometer Physica MCR502 (Anton Paar GmbH, Austria) using parallel plate geometry $20 \mathrm{~mm}$ in diameter. The sample suspension was injected between the plates and a magnetic field $\left(0-438 \mathrm{kA} \mathrm{m}^{-1}\right)$ perpendicular to the plates generated with a magnetic cell (Physica MRD 170+H-PTD200) was applied, while the gap between the plates was set to $0.5 \mathrm{~mm}$. The true magnetic field strength was determined with a Teslameter (Magnet-Physik, FH 51 , Dr Steingroever $\mathrm{GmbH}$, Germany). Shear rates $\left(10^{-2}\right.$ to $10^{2} \mathrm{~s}^{-1}$ ) were applied, and a shear stress and viscosity were determined in the absence (off-state) and in the presence (onstate) of a magnetic field. Prior to each on-state measurement,
MR suspensions were off-state sheared in order to disrupt possible residual structures. Then, new structures were induced using a given magnetic field strength. The temperature during testing was maintained at $25^{\circ} \mathrm{C}$.

\section{Results and discussion}

\section{Synthesis and characterization of PGMA-modified CI particles}

The surface modification of CI particles with PGMA chains was performed in several steps ${ }^{\mathbf{1 8}}$ as shown in Scheme 1. First, the oxidized CI surface was modified by reaction with an APTES silane agent. With this modifier it is also possible to make the grafted layer desirably stronger due to the horizontal crosslinking between modifier molecules. ${ }^{35}$ Subsequently, the introduced amine groups were modified by reaction with BiBB in order to introduce the ATRP initiator on to the CI surface. Finally, a surface-initiated ATRP of GMA was performed. In addition to ATRP initiator bonded on CI particles, a free sacrificial initiator was used in excess to decrease an extent of termination reactions due to a high concentration of active radicals on the CI surface and to allow better control of the molecular weight of PGMA. Two types of CI-PGMA particles varying in shell thickness were obtained by using two monomer/ initiator ratios leading to a modification of CI particles with two various molecular weights of PGMA.

The weight- and number-average molecular weights $\left(\bar{M}_{\mathrm{w}}\right.$, $\bar{M}_{\mathrm{n}}$ ) were determined by GPC analysis based on free PGMA chains grown from a free sacrificial initiator, under the assumption of the similar growth of the polymer from free and bond initiators. ${ }^{36}$ The results are summarized in Table 1. Based on the molecular weights of the grafted polymer chains it was

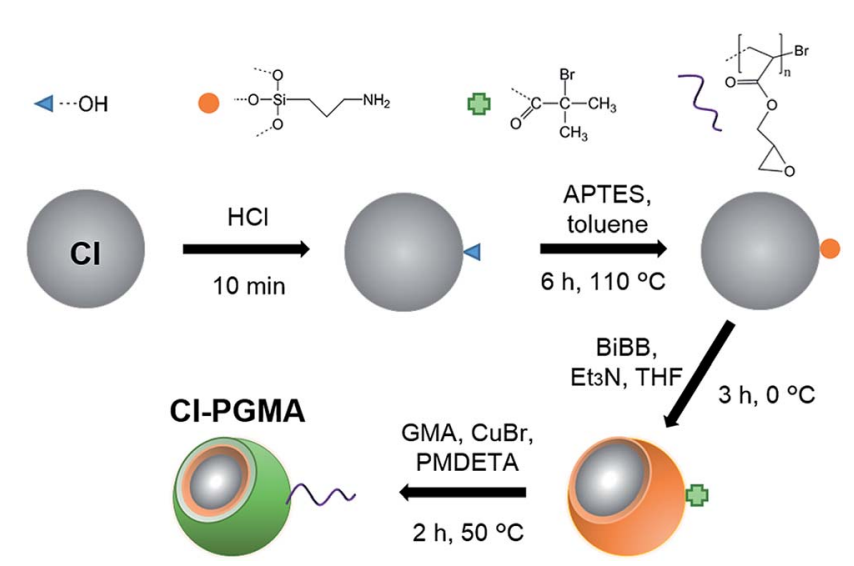

Scheme 1 Schematic picture of the synthesis of CI-PGMA particles.

Table 1 Weight- and number-average molecular weights and the dispersity index $\Theta$ of PGMA grafted onto $\mathrm{Cl}$ particles

\begin{tabular}{llll}
\hline Sample code & $\bar{M}_{\mathrm{w}}\left[\mathrm{g} \mathrm{mol}^{-1}\right]$ & $\bar{M}_{\mathrm{n}}[\mathrm{g} \mathrm{mol}$ & \\
\hline CI-PGMA-1 & 6600 & 5000 & $D[-]$ \\
CI-PGMA-2 & 12500 & 9700 & 1.32 \\
\end{tabular}


estimated theoretically that maximal lengths of fully stretched PGMA chains, and consequently shell thicknesses, are around 9 $\mathrm{nm}$ and $17 \mathrm{~nm}$ for the CI-PGMA-1 and CI-PGMA-2, respectively.

Fig. 1 shows SEM images of bare CI (a) and CI-PGMA-1 (b) particles. Particles possess a spherical shape with diameters under 5 microns. As can be seen, particles in both samples have comparable dimensions indicating negligible change in their size due to modification.

The modification of the CI surface was confirmed by EDS and FTIR analyses. In EDS measurements, the electron beams are only able to penetrate a few nanometers deep into the sample surface. ${ }^{37}$ This method was preferably employed for confirmation of the presence of characteristic elements in the PGMA layer. Comparing spectra of bare CI and CI-PGMA-1 particles, ${ }^{18}$ both of them showed strong Fe $\mathrm{L}, \mathrm{K} \alpha$, and $\mathrm{K} \beta$ lines; however, in CI-PGMA-1 particles, a higher content of carbon was determined, implying the presence of a PGMA layer on the surface of the CI particles. Successful CI surface modification was also indicated by substantial characteristic emission peaks, namely a $\mathrm{K} \alpha$ line of oxygen and a $\mathrm{K} \alpha$ line of silicon originated from the modifier.

In FTIR spectra of CI-PGMA- $1,{ }^{18}$ carbonyl $(\mathrm{C}=\mathrm{O})$ stretching vibrations and the asymmetric stretching of $\mathrm{C}-\mathrm{O}-\mathrm{C}$ from ester groups of PGMA were observed at $1714 \mathrm{~cm}^{-1}$ and $1350 \mathrm{~cm}^{-1}$, respectively. In addition, absorption peaks occurring at 904 $\mathrm{cm}^{-1}$ and $815 \mathrm{~cm}^{-1}$ were distinct indications of the presence of an oxirane ring.

The magnetic properties of the utilized particles were investigated. As shown in Fig. 1, the magnetic behavior of the coated particles was similar to that of the bare ones, but showed slightly lower values of magnetic moments due to a decreased magnetic susceptibility. However, the decreases of magnetizations in the modified particles were negligible in comparison to the bare ones (Fig. 2, inset A), as the thickness of the coatings was controlled via ATRP means. Magnetization curves were fitted with the J-A model, which further predicted slightly decreased values of saturation magnetizations. A value of $229 \mathrm{emu} \mathrm{g}^{-1}$ was reached for bare CI particles, while PGMAcoated variants exhibited magnetizations of $220 \mathrm{emu} \mathrm{g}^{-1}$ and $215 \mathrm{emu} \mathrm{g}^{-1}$, respectively. The values obtained by the application of the J-A equation were higher than those resulting from the experimental measurements, which might have occurred due to incomplete saturation magnetization as a result of the use of an insufficient magnetic field strength. ${ }^{38}$ Furthermore,
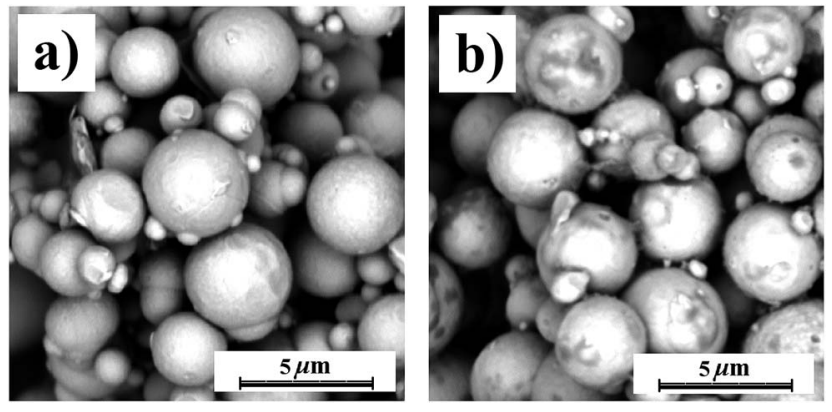

Fig. 1 SEM micrographs of bare $\mathrm{Cl}$ (a), and $\mathrm{Cl}$-PGMA-1 (b) particles.

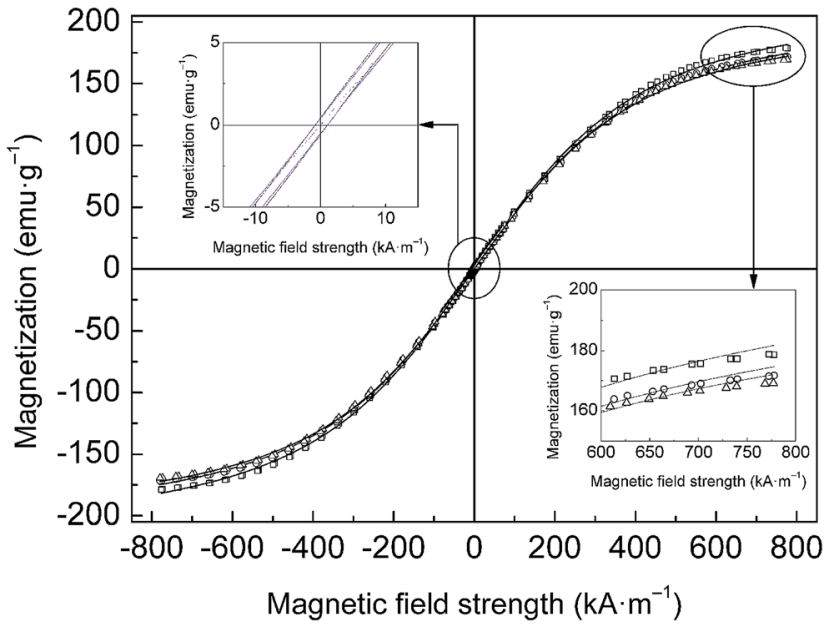

Fig. 2 VSM spectra of bare $\mathrm{Cl}$ (open squares), Cl-PGMA-1 (open circles), and $\mathrm{CI}$-PGMA-2 particles (open triangles). The solid lines represent the Jiles-Atherton model predictions.

the values of $\alpha$ parameter in the J-A model approached zero for all measurements, which indicated almost hysteresis-free curves (Fig. 2, inset B). The $A$ parameter in the J-A equation describing magnetization shape was calculated as $161 \mathrm{kA} \mathrm{m}^{-1}$ for bare CI particles, while values of $159 \mathrm{kA} \mathrm{m}^{-1}$ and $157 \mathrm{kA} \mathrm{m}^{-1}$ were obtained for CI-PGMA-1 particles and CI-PGMA-2 particles, respectively, which stem from a negligible change in the magnetic behavior of the CI particles as a consequence of the controlled surface modification.

Although the magnetic properties of the PGMA-modified particles were only slightly reduced compared to bare CI, their sedimentation stabilities in silicone oil were improved significantly due to enhanced interactions with the dispersion medium. This phenomenon was analyzed in more details in our previous paper. ${ }^{18}$

\section{Chemical stability}

Fig. 3 shows the $\mathrm{pH}$ value as a function of time for CI particles as well as CI-PGMA particles subjected to an acidic environment. In all measurements, the $\mathrm{pH}$ value initially decreases, which might be caused by local sample stabilization in the vicinity of the pH-meter probe. The experiment is based on the reaction of an aqueous $\mathrm{HCl}$ solution with $\mathrm{Fe}$. The oxidation of Fe proceeds according to the equation (ESI $\dagger$ ).

Shortly after the initiation of the experiment, the $\mathrm{pH}$ value of the dispersion containing bare CI particles exhibited a gradual increase accompanied by an intensive $\mathrm{H}_{2}$ bubble evolution. Approximately after 40 minutes, the leaking amount of $\mathrm{H}_{2}$ decreased, while an ongoing increase of the $\mathrm{pH}$ value was observed.

On the contrary, both types of CI-PGMA particles demonstrated excellent anti-acid corrosion stability. The PGMA shell and its APTES grafting base covered the CI core, and the reaction almost did not proceed. However, minimal production of $\mathrm{H}_{2}$ was observed, which implied that the APTES base was not fully compact, and thus the chloride ions were still able to react 


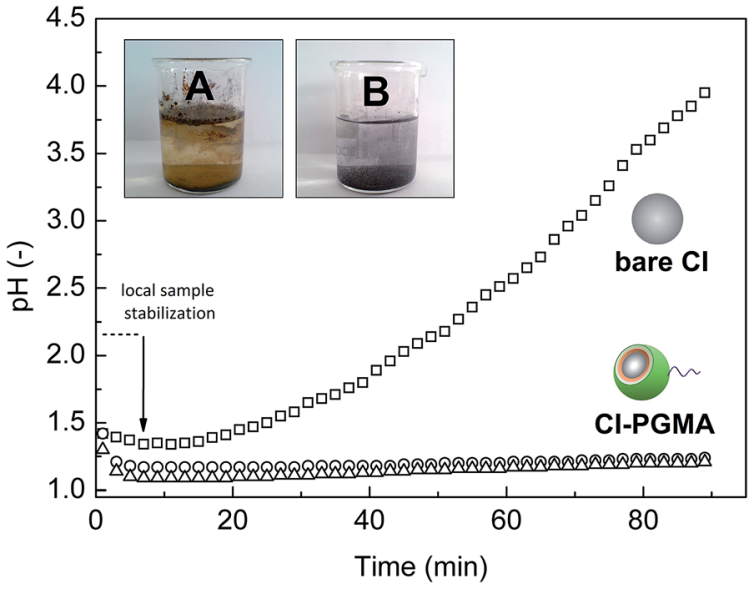

Fig. 3 Resistance of bare $\mathrm{Cl}$ particles (open squares), Cl-PGMA-1 particles (open circles), and $\mathrm{Cl}-\mathrm{PGMA}-2$ particles (open triangles) against an acidic environment $(0.05 \mathrm{M} \mathrm{HCl})$. The inset image shows a tremendous difference in chemical stability between bare $\mathrm{Cl}$ particles (A) and $\mathrm{Cl}$-PGMA-1 particles (B) after a 36 hour exposure.

with the Fe core. Nevertheless, compared to bare CI the improvement in chemical stability was significant. Generally, the $\mathrm{pH}$ remained at a similar value throughout the whole experiment. It was also clear that for the purpose of chemical stability enhancement, it was sufficient to modify the CI particles with PGMA chains with a molecular weight of $6600 \mathrm{~g} \mathrm{~mol}^{-1}$, since no further improvement of chemical stability occurred with the increasing molecular weight of grafted PGMA.

After a 90 minute exposure, a difference of 3 orders on the $\mathrm{pH}$ scale was reached, and the measurement was stopped. However, the samples were left in solutions. The inset of Fig. 3 shows that the modified particles were still in a very good condition after a 36 hour exposure. Hence, the results of the stability test showed that CI-PGMA particles are very promising candidates for practical implementation.

Magnetic particles have also found promising applicability in the biomedical field. Cell therapy (cell separation and labeling), ${ }^{25}$ cancer therapy, ${ }^{26}$ and particularly drug delivery ${ }^{27}$ have been examined using such remarkable structures. It was shown that in order to target organs that lie deeply in the body cavity (8-12 $\mathrm{cm}$ from the body surface), the use of magnetic microparticles $(0.5-5 \mu \mathrm{m})$ is necessary. Besides that, PGMA has recently gained a special interest in drug and biomolecule binding where the presence of an epoxy ring represents an ideal site for a coupling reaction with pharmaceuticals. ${ }^{29,39}$

With this knowledge, PGMA was deliberately chosen as a shell material for the ATRP coating of CI particles. Prepared CI-PGMA particles may represent a promising material for drug delivery to organs deep within the body. ${ }^{28}$ Thus, in this article the cytotoxicity of prepared particles as an important characteristic was further studied.

\section{Cytotoxicity}

The results performed on the NIH/3T3 cell line have shown that tested concentrations of extracts have not distinctly affected the cell viability. The cytotoxicity of bare CI particles belongs to a category with an absence of cytotoxicity within the whole tested concentration range. Nevertheless, the primary interest of this study was to investigate the cytotoxicity of CI-PGMA particles. The maximum loss of cell viability was observed in the $75 \%$ extract, where 0.85 cell survival was reached, which is still related to the absence of cytotoxicity. For all other concentrations of extracts, namely $50 \%, 25 \%, 10 \%$, and $1 \%$, the absence of cytotoxicity was more obvious, whereas the values of viability in these concentrations were around 1.00 compared to the reference.

Results of the MTT assay were also confirmed by microscopic observations. Fig. 4 shows the effect of extract concentration on changes in cell morphologies. The absence of cytotoxicity of CI-PGMA particles and their gained functionality make them very interesting from a further research point of view, as the appropriate substances can be attached. Considering the observations (Table 2), it can be asserted that CI-PGMA particles have the potential to be successfully used in medicine.

\section{Rheological measurements}

The steady shear flow properties of prepared silicone oil MR suspensions were measured in a controlled shear rate mode. The collected data was properly investigated in order to describe the change of rheological behavior upon the application of the external magnetic field. Fig. 5 presents shear stresses of prepared MR suspensions as a function of the shear rates under

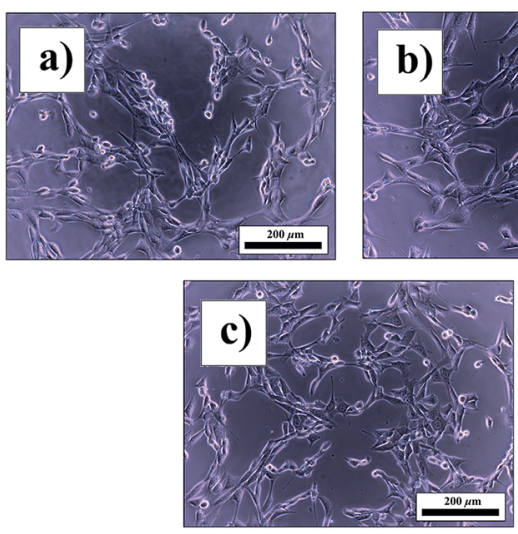

Fig. 4 Micrographs of the NIH/3T3 mouse embryonic fibroblast cell line, ATCC CRL-1658 treated with (a) 50\% Cl extract, (b) 50\% Cl-PGMA extract, and (c) reference.

Table 2 The cytotoxicity of $\mathrm{Cl}$ and $\mathrm{Cl}$-PGMA extracts of various concentrations on the $\mathrm{NIH} / 3 \mathrm{~T} 3$ cell line reported according to the requirements of EN ISO 10993-5 standard

\begin{tabular}{lllll}
\hline Extract & Control & NIH/3T3, 1.00 & Control & NIH/3T3, 1.00 \\
\hline $75 \%$ & CI & 0.97 & CI-PGMA & 0.85 \\
$50 \%$ & & 0.93 & & 0.98 \\
$25 \%$ & & 0.99 & & 1.01 \\
$10 \%$ & & 0.95 & & 1.02 \\
$1 \%$ & & 0.95 & & 0.98
\end{tabular}




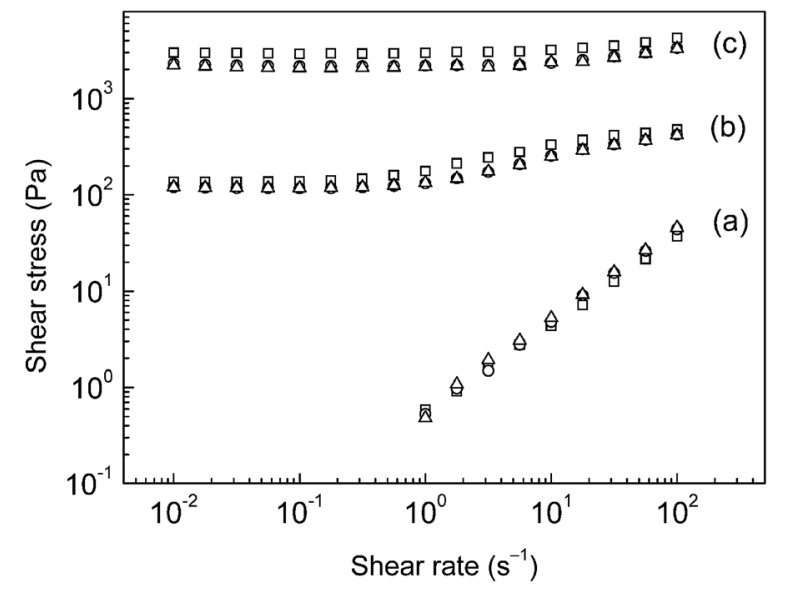

Fig. 5 The dependence of the shear stress on the shear rate for a $40 \mathrm{wt} \%$ suspension of $\mathrm{Cl}$ particles (open squares), CI-PGMA-1 particles (open circles), and Cl-PGMA-2 particles (open triangles) in silicone oil under $0 \mathrm{kA} \mathrm{m}^{-1}$ (a), $87 \mathrm{kA} \mathrm{m}^{-1}$ (b), and $438 \mathrm{kA} \mathrm{m}^{-1}$ (c) magnetic field strengths.

various magnetic field strengths. The shear stresses of suspensions based on CI-PGMA particles exhibit similar values when compared to the MR suspension of bare CI, which proves a promising MR performance of the prepared materials. As can be seen, in spite of a relatively low particle loading, the shear stresses increased almost over four orders of magnitude by the application of a $438 \mathrm{kA} \mathrm{m}^{-1}$ magnetic field strength in comparison to the off-state behavior at lower shear rates, whereas a shear stress increase by two orders of magnitude was attained at higher shear rates. The on-state behavior can be characterized by the wide shear stress plateau within the shear rate range, which is attributed to the formation of internal chain-like structures resulting in the solid-like character of MR suspensions. ${ }^{40}$

In order to exclude possible data distortion, the off-state flow curves are not shown within the whole shear rate range due to the inappropriateness of measuring MR geometry PP20 for low shear rates as, was also presented by authors. ${ }^{41}$

Flow curves in linear scaling fitted with the HB model (eqn (3)) are depicted in Fig. 6. The displayed rheological data correspond to the MR suspension based on bare CI particles under various magnetic field strengths. Rheograms of MR suspensions containing modified particles are included in ESI (please see Fig. S1†). As can be observed, the off-state behavior of the MR suspension can be denoted as Newtonian, however, in the on-state, the MR suspension exhibits a non-linear increase of shear stress with an increasing shear rate, which can be characterized as non-Newtonian shear thinning behavior. As initially expected, shear stress increased with increasing magnetic field strength due to a more pronounced chain-like formation. A similar behavior was also observed in suspensions of PGMA-coated particles in which, however, shear stresses exhibited slightly lower values. The obtained experimental data was analyzed using the HB model, which is expressed as follows:

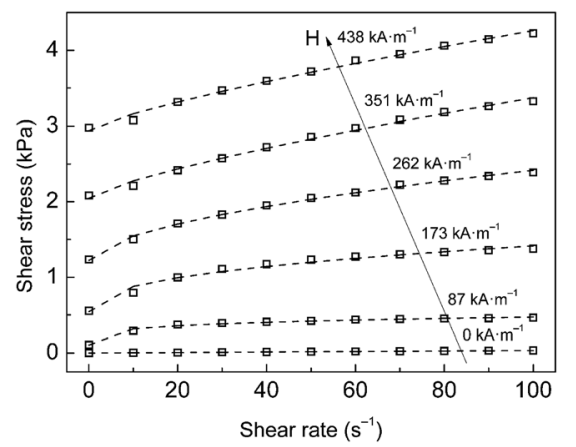

Fig. 6 Rheograms of $40 \mathrm{wt} \% \mathrm{MR}$ suspension of bare $\mathrm{Cl}$ particles showing experimental data (open squares) fitted with the HB model (dashed lines) under various magnetic field strengths.

$$
\left.\begin{array}{rl}
\tau=\tau_{0} \operatorname{sgn}(\dot{\gamma})+K \dot{\gamma}^{n}, & |\tau|>\tau_{0} \\
\dot{\gamma}=0, & |\tau|<\tau_{0}
\end{array}\right\}
$$

where $\tau$ is the shear stress, $\tau_{0}$ means the dynamic yield stress controlled by the magnetic field strength, sgn denotes the signum function, $\dot{\gamma}$ represents shear rate, $K$ and $n$ are the consistency index and the power-law exponent, respectively. The HB model provided a reasonably good fit with the experimental data, as was further proved by statistical evaluation (Table S1) (ESI $\dagger$ ).

The dynamic yield stresses predicted according to the HB model (Fig. 7) are plotted as a function of applied magnetic field strength. Surface modification reduced the magnetic susceptibility of the particles. Therefore, the yield stresses of MR suspensions containing coated particles were lower; however, due to controllable coating the decrease was insignificant. Nevertheless, the increase in the molecular weight of the polymer coating layer caused a further decrease in the yield stress (Table 3).

The displayed dependences exhibit similar patterns, as were described previously by authors. ${ }^{42,43}$ According to numerical and mathematical models, the dynamic yield stress varies with $\mathrm{H}^{2-1.5}$ depending on a mechanism of the polarization. The behavior of the MR suspensions follows a dipole mechanism at relatively low

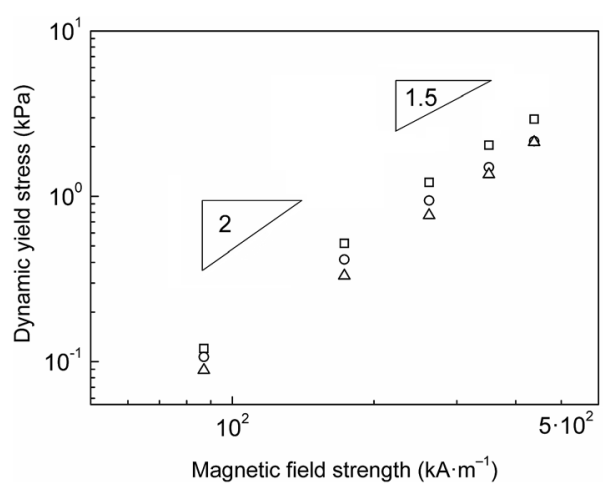

Fig. 7 Predicted dynamic yield stresses of MR suspensions based on bare $\mathrm{Cl}$ particles (open squares), Cl-PGMA-1 particles (open circles), and $\mathrm{Cl}-\mathrm{PGMA}-2$ particles (open triangles) as a function of magnetic field strength. 
Table 3 Summary of yield stress values under various magnetic field strengths

Dynamic yield stress $[\mathrm{kPa}]$ at certain magnetic field strengths

\begin{tabular}{|c|c|c|c|c|c|}
\hline Dispersed phase & $87 \mathrm{kA} \mathrm{m}^{-1}$ & $173 \mathrm{kA} \mathrm{m}^{-1}$ & $262 \mathrm{kA} \mathrm{m}^{-1}$ & $351 \mathrm{kA} \mathrm{m}^{-1}$ & $438 \mathrm{kA} \mathrm{m}^{-1}$ \\
\hline Bare CI & 0.120 & 0.519 & 1.215 & 2.046 & 2.941 \\
\hline CI-PGMA-1 & 0.107 & 0.417 & 0.944 & 1.503 & 2.160 \\
\hline
\end{tabular}

magnetic fields, which is reflected in the quadratic dependence of the yield stress on the magnetic field strength. At higher magnetic fields, local saturation magnetization of the particles arises, and the yield stress further varies only with $\mathrm{H}^{1.5}$. Nevertheless, the noticeable change of the slope in Fig. 7 was apparent only in the MR suspension of bare CI particles. This behavior can be associated with exceeding the critical magnetic field strength. MR suspensions of both variants of PGMA-coated particles exhibited an almost linear trend within the whole magnetic field strength range, which can be attributed to the presence of the PGMA shell affecting the contact zones between the particles.

\section{Evaluation of MR efficiency}

As for electrorheological suspensions, ${ }^{44}$ the performance of MR suspensions can be in some instances described with MR efficiencies rather than absolute values of shear stresses. MR efficiency $(e)$ is defined for any given $\dot{\gamma}$ as:

$$
e=\frac{\left(\eta_{\mathrm{E}}-\eta_{0}\right)}{\eta_{0}}
$$

where $\eta_{\mathrm{E}}$ represents the on-state viscosity of the MR suspension and $\eta_{0}$ denotes the off-state the viscosity at the same $\dot{\gamma}$.

Fig. 8 illustrates $e$ as a function of applied magnetic field strength at $\dot{\gamma}=1 \mathrm{~s}^{-1}$. The highest values of $e$ obviously demonstrate the MR suspension of bare CI particles. MR suspensions of PGMA-coated particles exhibit a similar trend, nevertheless, with lower values of $e$. The molecular weight of the polymer shell appears to have a negligible effect on $e$ in this case.

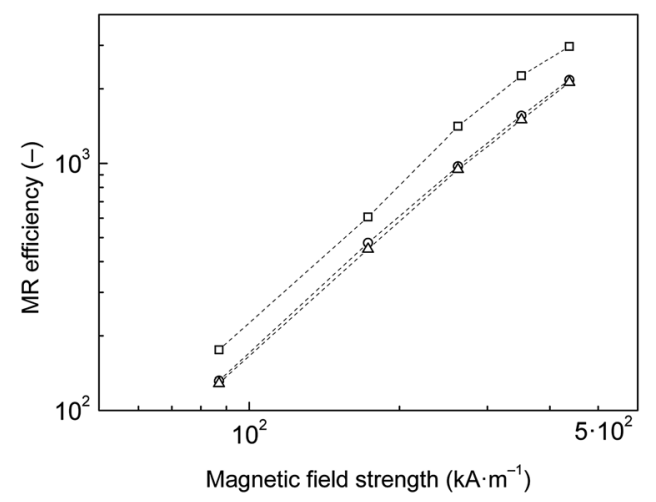

Fig. 8 The dependence of MR efficiency on the applied magnetic field strength for MR suspensions containing $40 \mathrm{wt} \%$ of $\mathrm{Cl}$ particles (open squares), CI-PGMA-1 particles (open circles), and CI-PGMA-2 particles (open triangles) in silicone oil at $1 \mathrm{~s}^{-1}$.

\section{Conclusion}

Two types of CI particles grafted with PGMA of different thicknesses were synthesized using a surface-initiated ATRP. It has been found that a PGMA coating layer provides excellent antiacid corrosion properties. No significant difference in particle chemical stability was observed as the molecular weight of grafted PGMA increased from 6600 to $12500 \mathrm{~g} \mathrm{~mol}^{-1}$. Secondly, the in vitro cytotoxicity test conducted according to ISO standards shows that CI-PGMA particles are not cytotoxic. The absence of cytotoxicity, the presence of an easily transformable epoxy ring, and a high magnetic response make the prepared CI-PGMA particles a promising material for biomedical applications, especially for drug delivery to organs deep within the body.

The rheological properties of silicon oil-based MR suspensions were evaluated over a magnetic field strength range using the HB model and provided a reasonably good fit with the experimental data, as was supported by statistical evaluation (ESI). MR suspensions of PGMA-coated CI particles generated sufficient dynamic yield stresses compared to the suspension of bare CI particles. However, it was shown that the lower molecular weight of the PGMA was reflected in less suppressed MR activity. In general, yield stresses of the MR suspensions were quadratically dependent on the applied magnetic field strength. Assuming these and previously described findings to be accurate, it can be concluded that CI-PGMA particles obtained using surface-initiated ATRP appear to be a versatile material for miscellaneous applications.

\section{Author contributions}

The manuscript was contributed to by all authors, and all authors have approved the final version of the manuscript.

\section{Acknowledgements}

This article was written with support of the Operational Program Research and Development for Innovations co-funded by the European Regional Development Fund (ERDF) and the National Budget of the Czech Republic, within the framework of the project Centre of Polymer Systems (CZ.1.05/2.1.00/03.0111). The author M. C. wishes to thank the internal grant of TBU in Zlin No. IGA/CPS/2015/007 funded from specific university research resources. Authors M. I. and J. M. appreciate the financial support of the Centre of Excellence FUN-MAT. Z. K. 
and P. H. further thank the Czech Science Foundation (1308944S) for financial support.

\section{References}

1 B. J. Park, F. F. Fang and H. J. Choi, Magnetorheology: materials and application, Soft Matter, 2010, 6, 5246-5253.

2 M. Mrlik, M. Sedlacik, V. Pavlinek, P. Bazant, P. Saha, P. Peer and P. Filip, Synthesis and magnetorheological characteristics of ribbon-like, polypyrrole-coated carbonyl iron suspensions under oscillatory shear: materials and application, J. Appl. Polym. Sci., 2013, 128, 2977-2982.

3 A. J. F. Bombard, M. Knobel and M. R. Alcantara, Phosphate coating on the surface of carbonyl iron powder and its effect in magnetorheological suspensions, Int. J. Mod. Phys. B, 2007, 21, 4858-4867.

4 F. F. Fang, Y. D. Liu, H. J. Choi and Y. Seo, Core-shell structured carbonyl iron microspheres prepared via dualstep functionality coatings and their magnetorheological response, ACS Appl. Mater. Interfaces, 2011, 3, 3487-3495.

5 J. de Vicente, D. J. Klingenberg and R. Hidalgo-Alvarez, Magnetorheological fluids: a review, Soft Matter, 2011, 7, 3701-3710.

6 G. Bossis, S. Lacis, A. Meunier and O. Volkova, Magnetorheological fluids, J. Magn. Magn. Mater., 2002, 252, 224-228.

7 M. Sedlacik, V. Pavlinek, P. Saha, P. Svrcinova, P. Filip and J. Stejskal, Rheological properties of magnetorheological suspensions based on core-shell structured polyanilinecoated carbonyl iron particles, Smart Mater. Struct., 2010, 19, 115008.

8 G. Yang, B. F. Spencer, J. D. Carlson and M. K. Sain, Largescale MR fluid dampers: modeling and dynamic performance considerations, Eng. Struct., 2002, 24, 309-323.

9 Y. Ding, L. Zhang, H. T. Zhu and Z. X. Li, A new magnetorheological damper for seismic control, Smart Mater. Struct., 2013, 22, 115003.

10 D. H. Wang and W. H. Liao, Magnetorheological fluid dampers: a review of parametric modelling, Smart Mater. Struct., 2011, 20, 023001.

11 S. Dai, C. Du and G. Yu, Design, testing and analysis of a novel composite magnetorheological fluid clutch, J. Intell. Mater. Syst. Struct., 2013, 24, 1675-1682.

12 W. Kordonski and A. Shorey, Magnetorheological (MR) Jet Finishing Technology, J. Intell. Mater. Syst. Struct., 2007, 18, 1127-1130.

13 T. Kikuchi, K. Oda and J. Furusho, Leg-robot for demonstration of spastic movements of brain-injured patients with compact magnetorheological fluid clutch, Adv. Robot., 2010, 24, 671-686.

14 M. Sedlacik, V. Pavlinek, M. Lehocky, A. Mracek, O. Grulich, P. Svrcinova, P. Filip and A. Vesel, Plasma-treated carbonyl iron particles as a dispersed phase in magnetorheological fluids, Colloids Surf., A, 2011, 387, 99-103.

15 X. Quan, W. Chuah, Y. Seo and H. J. Choi, Core-shell structured polystyrene coated carbonyl iron microspheres and their magnetorheology, IEEE Trans. Magn., 2014, 50, 1-4.
16 M. Mrlik, M. Ilcikova, M. Sedlacik, J. Mosnacek, P. Peer and P. Filip, Cholesteryl-coated carbonyl iron particles with improved anti-corrosion stability and their viscoelastic behaviour under magnetic field, Colloid Polym. Sci., 2014, 292, 2137-2143.

17 K. Matyjaszewski, Atom transfer radical polymerization (ATRP): Current status and future perspectives, Macromolecules, 2012, 45, 4015-4039.

18 M. Cvek, M. Mrlik, M. Ilcikova, T. Plachy, M. Sedlacik, J. Mosnacek and V. Pavlinek, A facile controllable coating of carbonyl iron particles with poly(glycidyl methacrylate): a tool for adjusting MR response and stability properties, $J$. Mater. Chem. C, 2015, 3, 4646-4656.

19 J. D. Carlson and M. R. Jolly, MR fluid, foam and elastomer devices, Mechatronics, 2000, 10, 555-569.

20 V. C. Kelessidis and R. Maglione, Modeling rheological behavior of bentonite suspensions as Casson and Robertson-Stiff fluids using Newtonian and true shear rates in Couette viscometry, Powder Technol., 2006, 168, 134-147.

$21 \mathrm{M}$. Yu, S. Wang, J. Fu and Y. Peng, Unsteady analysis for oscillatory flow of magnetorheological fluid dampers based on Bingham plastic and Herschel-Bulkley models, J. Intell. Mater. Syst. Struct., 2013, 24, 1067-1078.

22 F. Ahmadkhanlou, M. Mahboob, S. Bechtel and G. Washington, An improved model for magnetorheological fluid-based actuators and sensors, J. Intell. Mater. Syst. Struct., 2010, 21, 3-18.

23 A. Sidpara, M. Das and V. K. Jain, Rheological characterization of magnetorheological finishing fluid, Mater. Manuf. Processes, 2009, 24, 1467-1478.

24 S. D. Jacobs, A. Duparre and R. Geyl, MRF with adjustable pH, Optical Fabrication, Testing, and Metrology IV, 2011, 8169, 816902.

25 J. L. Arias, V. Gallardo, F. Linares-Molinero and A. V. Delgado, Preparation and characterization of carbonyl iron/ poly(butylcyanoacrylate) core/shell nanoparticles, J. Colloid Interface Sci., 2006, 299, 599-607.

26 G. A. Flores and J. Liu, Embolization of blood vessels as a cancer therapy using magnetorheological fluids, J. Intell. Mater. Syst. Struct., 2002, 13, 641-646.

27 A. K. A. Silva, E. L. Silva, A. S. Carrico and E. S. T. Egito, Magnetic carriers: a promising device for targeting drugs into the human body, Curr. Pharm. Des., 2007, 12, 1179.

28 F. Akai, M. Maeda, S. Hashimoto, M. Taneda and H. Takagi, A new animal model of cerebral infarction: magnetic embolization with carbonyl iron particles, Neurosci. Lett., 1995, 194, 139-141.

29 P. F. Canamero, J. L. de la Fuente, E. L. Madruga and M. Fernandez-Garcia, Atom transfer radical polymerization of glycidyl methacrylate: a functional monomer, Macromol. Chem. Phys., 2004, 205, 2221-2228.

30 F. Liorzou, B. Phelps and D. L. Atherton, Macroscopic models of magnetization, IEEE Trans. Magn., 2000, 36, 418-428.

31 P. Humpolicek, V. Kasparkova, P. Saha and J. Stejskal, Biocompatibility of polyaniline, Synth. Met., 2012, 162, 722-727. 
32 C. L. Bayer, I. J. Trenchard and N. A. Peppas, Analyzing polyaniline-poly(2-acrylamido-2-methylpropane sulfonic acid) biocompatibility with $3 \mathrm{~T} 3$ fibroblasts, J. Biomater. Sci., Polym. Ed., 2010, 21, 623-634.

33 J. Stejskal, M. Hajna, V. Kasparkova, P. Humpolicek, A. Zhigunov and M. Trchova, Purification of a conducting polymer, polyaniline, for biomedical applications, Synth. Met., 2014, 195, 286-293.

34 M. Bosetti, A. Masse, E. Tobin and M. Cannas, Silver coated materials for external fixation devices: in vitro biocompatibility and genotoxicity, Biomaterials, 2002, 23, 887-892.

35 S. G. Belyavskii, P. G. Mingalyov, F. Giulieri, R. Combarrieau and G. V. Lisichkin, Chemical modification of the surface of a carbonyl iron powder, Prot. Met., 2006, 42, 244-252.

36 G. Goncalves, P. Marques, A. Barros-Timmons, I. Bdkin, M. K. Singh, N. Emami and J. Gracio, Graphene oxide modified with PMMA via ATRP as a reinforcement filler, $J$. Mater. Chem., 2010, 20, 9927-9934.

37 J. Sutrisno, A. Fuchs, H. Sahin and F. Gordaninejad, Surface coated iron particles via atom transfer radical polymerization for thermal-oxidatively stable high viscosity magnetorheological fluid, J. Appl. Polym. Sci., 2013, 128, 470-480.

38 M. Sedlacik, V. Pavlinek, R. Vyroubal, P. Peer and P. Filip, A dimorphic magnetorheological fluid with improved oxidation and chemical stability under oscillatory shear, Smart Mater. Struct., 2013, 22, 035011.

39 C. Shanthi and K. P. Rao, Chitosan modified poly(glycidyl methacrylate-butyl acrylate) copolymer grafted bovine pericardial tissue-anticalcification properties, Carbohydr. Polym., 2001, 44, 123-131.

40 S. Y. Kim, S. H. Kwon, Y. D. Liu, J. S. Lee, C. Y. You and H. J. Choi, Core-shell-structured cross-linked poly(glycidyl methacrylate)-coated carbonyl iron microspheres and their magnetorheology, J. Mater. Sci., 2014, 49, 1345-1352.

41 M. S. Cho, H. J. Choi and M. S. Jhon, Shear stress analysis of a semiconducting polymer based electrorheological fluid system, Polymer, 2005, 46, 11484-11488.

42 F. F. Fang, H. J. Choi and M. S. Jhon, Magnetorheology of soft magnetic carbonyl iron suspension with single-walled carbon nanotube additive and its yield stress scaling function, Colloids Surf., A, 2009, 351, 46-51.

43 J. M. Ginder, L. C. Davis and L. D. Elie, Rheology of magnetorheological fluids: Models and measurements, Int. J. Mod. Phys. B, 1996, 10, 3293-3303.

44 M. Machovsky, M. Mrlik, I. Kuritka, V. Pavlinek and V. Babayan, Novel synthesis of core-shell urchin-like ZnO coated carbonyl iron microparticles and their magnetorheological activity, RSC Adv., 2013, 4, 996-1003. 